\title{
The GAMA Panchromatic Survey
}

\author{
Simon P. Driver ${ }^{1,2}$ \\ ${ }^{1}$ International Centre for Radio Astronomy Research, University of Western Australia, \\ Crawley, WA 6009, Australia, \\ ${ }^{2}$ Scottish Universities Physics Alliance (SUPA), School of Physics and Astronomy, University \\ of St Andrews, St Andrews, Scotland
}

\begin{abstract}
The Galaxy And Mass Assembly Survey (GAMA) has now been operating for almost 5 years gathering spectroscopic redshifts for five regions of sky spanning 300 sq degrees in total to a depth of $r<19.8$ mag. The survey has amassed over 225,000 redshifts making it the third largest redshift campaign after the SDSS and BOSS surveys. The survey has two novel features that set it apart: (1) complete and uniform sampling to a fixed flux limit $(r<19.8 \mathrm{mag})$ regardless of galaxy clustering due to multiple-visits to each sky region, enabling the construction of high-fidelity catalogues of groups and pairs, (2) co-ordination with diverse imaging campaigns which together sample an extremely broad range along the electro-magnetic spectrum from the UV (GALEX) through optical (VST KIDs), near-IR (VISTA VIKING), mid-IR (WISE), far-IR (Herschel-Atlas), $1 \mathrm{~m}$ (GMRT), and eventually $20 \mathrm{~cm}$ continuum and rest-frame $21 \mathrm{~cm}$ line measurements (ASKAP DINGO). Apart from the ASKAP campaign all multi-wavelength programmes are either complete or in the final stages of observations and the UV-far-IR data are expected to be fully merged by the end of 2013. This article provides a brief flavour of the coming panchromatic database which will eventually include measurements or upper-limits across 27 wavebands for 380,000 galaxies. GAMA DR2 is scheduled for the end of January 2013.
\end{abstract}

\section{Introduction}

The Galaxy And Mass Assembly Survey (GAMA; Driver et al. 2009, Driver et al. 2011) will contain spectroscopic redshifts at $>98 \%$ completeness for 380,000 galaxies drawn from five distinct sky regions $\left(2^{h}, 9^{h}, 12^{h}, 15^{h}\right.$, and $\left.23^{h}\right)$. Details of the survey concept, input catalogue, tiling strategy and first data release are provided in Driver et al. (2009), Baldry et al. (2010), Robotham et al. (2010), and Driver et al. (2011) respectively. The construction of the first group catalogue is given by Robotham et al. (2011) and the measurement of robust stellar masses and Sérsic profiles by Taylor et al. (2011) and Kelvin et al. (2012) respectively. Current early science includes the measurement of the nearby galaxy luminosity functions from $U V-K$ Driver et al. (2012a) and their evolution in ugriz to $z<0.5$ by Loveday et al. (2012). The nearby galaxy stellar mass function, which exhibits a distinctive upturn at intermediate masses, is given by Baldry et al. (2012) and upcoming papers by Taylor et al. (2013), Lopez-Cruz et al. (2013), Gunawardhana et al. (2013) and Robotham et al. (2013) explore galaxy colour bimodality, the evolution of the mass-metallicity relation, the evolution of the $\mathrm{H} \alpha$ luminosity function, and the life and times of $\mathrm{L}^{*}$ galaxies. Numerous $(>100)$ other papers are currently in progress. Fig. 1 shows the end-of-year 2012 update to the survey of surveys table given in Driver (2012b) and provides some indication of GAMAs emerging status amongst established leading surveys from the past three decades. Modern surveys like GAMA, BOSS and VIPERS are all expected to rise rapidly up this league table over the coming years and updates will be reported annually. 
Table 1. Major extragalactic optical/near-IR surveys and their impact in terms of refereed papers and citations

\begin{tabular}{|c|c|c|}
\hline Survey & | Papers & Citations \\
\hline Sloan Digital Sky Survey (SDSS) & 3803 & 163288 \\
\hline Hubble Deep Field (HDF) & 594 & 49360 \\
\hline Two Micron All Sky Survey (2MASS) & 1076 & 32506 \\
\hline Great Observatories Origins Deep Survey (GOODS) & 567 & 30331 \\
\hline Two degree Field Galaxy Redshift Survey (2dFGRS) & 206 & 25301 \\
\hline Centre for Astrophysics (CfA) & 342 & 19883 \\
\hline Galaxy Evolution Explorer (GALEX) & 563 & 15532 \\
\hline Cosmic Evolution Survey (COSMOS) & 394 & 13060 \\
\hline Ultra Deep Field (UDF) & 202 & 9169 \\
\hline Canada France Redshift Survey (CFRS) & 90 & 8797 \\
\hline Deep Evolutionary Exploratory Probe (DEEP/DEEP2) & 154 & 7767 \\
\hline Classifying Objects by Medium-Band Observations (COMBO-17/COMBO17) & 89 & 6882 \\
\hline UKIRT Infrared Deep Sky Survey (UKIDSS) & 135 & 4661 \\
\hline VIMOS VLT Deep Survey (VVDS) & 83 & 4531 \\
\hline Las Campanas Redshift Survey (LCRS) & 96 & 4197 \\
\hline Southern Sky Redshift Survey (SSRS/SSRS2) & 89 & 4151 \\
\hline APM Galaxy Survey (APMGS) & 57 & 4109 \\
\hline Point Source Catalogue Survey (PSCz) & 95 & 3553 \\
\hline $2 \mathrm{dF}$ QSO redshift Survey (2QZ) & 57 & 3305 \\
\hline $\begin{array}{l}\text { Canadian Network for Observational Cosmology Field Galaxy Redshift } \\
\text { Survey (CNOC2) }\end{array}$ & 65 & 3169 \\
\hline CFHT Legacy Survey (CFHTLS) & 87 & 3151 \\
\hline Galaxy Evolution from Morphologies and SEDs (GEMs) & 68 & 3109 \\
\hline NOAO Deep Wide Field Survey (NDFWS) & 76 & 2772 \\
\hline zCOSMOS & 60 & 2218 \\
\hline Millennium Galaxy Catalogue (MGC) & 38 & 1854 \\
\hline The 2dF-SDSS LRG And QSO Survey (2SLAQ) & 36 & 1636 \\
\hline AGN and Galaxy Evolution Survey (AGES) & 34 & 1575 \\
\hline Gemini Deep Deep Survey (GDDS) & 20 & 1479 \\
\hline Stromlo-APM Redshift Survey (SARS) & 19 & 1402 \\
\hline Baryonic Acoustic Oscillations Survey (BOSS) & 45 & 980 \\
\hline ESO Slice Project (ESP) & 24 & 971 \\
\hline The $6 \mathrm{dF}$ Galaxy Survey (6dFGS) & 26 & 749 \\
\hline WiggleZ & 26 & 646 \\
\hline Galaxy And Mass Assembly (GAMA) & 33 & 468 \\
\hline 2MASS Redshift Survey (2MRS) & 19 & 413 \\
\hline VIMOS Public Extragalactic Redshift Survey (V & 3 & 3 \\
\hline
\end{tabular}

Note these numbers were determined on $28^{\text {th }}$ Dec 2012 using the SOA/NASA ADS Astronomy Query Form by searching for refereed papers which contained abstract keywords based on the following boolean logic: (galaxy or galaxies) and (" $<$ long survey name $>$ " or $<$ short survey name $>$ ). The table is purely indicative and not weighted by survey age or effective cost and is updated on an annual basis. My apologies in advance for the many surveys not included.

\section{The GAMA panchromatic campaign}

As stated earlier a number of major imaging surveys are underway on a variety of internationally leading facilities which include within their survey footprints the GAMA sky regions. These complimentary imaging surveys are a mixture of GAMA motivated/led programs (e.g., GALEX, GMRT), those in which GAMA team members are playing a leading role (e.g., VISTA VIKING, ASKAP DINGO), to those in which GAMA is peripheral or playing a supporting role (e.g., VST KIDs, WISE, Herschel-Atlas). Regardless of the motivation these data collectively form the GAMA panchromatic campaign and need to be assembled, astrometrically matched, and for photometric measurements to be conducted which manages highly variable flux limits and spatial resolutions. Typically only $10-20 \%$ of the GAMA galaxies have clear unambiguous counterparts in all bands and spatial resolutions which vary with wavelength from $0.5^{\prime \prime}$ to $30^{\prime \prime}$. The significance of managing these two issues (flux limits and spatial resolutions), cannot be overstated. The correct strategy to manage this process is not yet entirely clear, the wrong strategy 

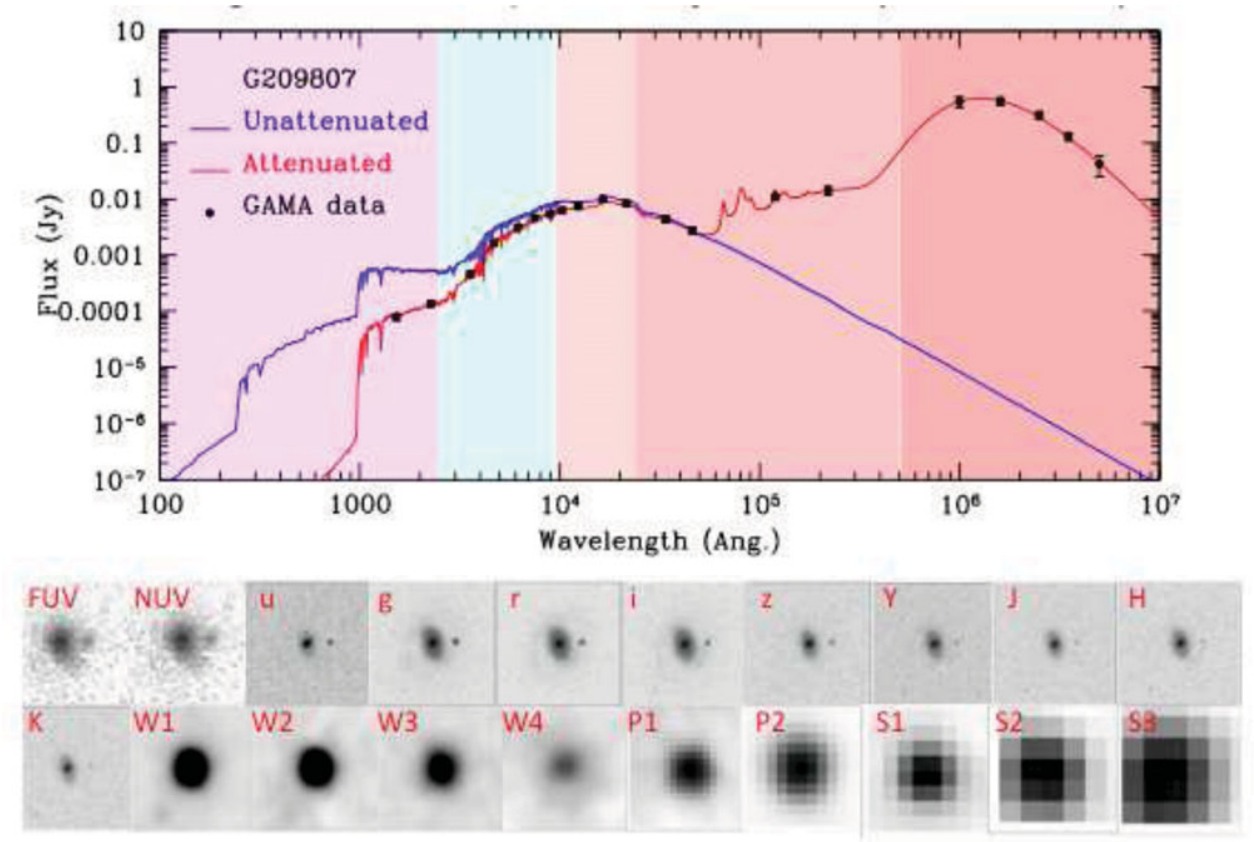

Figure 1. (upper plot) MAGPHYS modelling of a single GAMA galaxy detected in GALEX, SDSS, UKIDSS, WISE, HERSCHEL PACS and HERSCHEL SPIRE. (lower panels) The postage stamps images are shown in the lower panels highlighting the varied spatial resolutions of the contributing surveys.

however is. To simply conduct independent source finding in each band and then attempt to table match the resulting catalogues leads to a highly biased (ill defined) sample with high levels of blended contamination in the poorer resolution bands. The problem is particularly acute when matching data from the optical to the far-IR where the incidence of high-z interlopers and lensed sources becomes a significant problem. Fig. 1 highlights some of these issues by showing the assembled data for a single object which is clearly detected and unambiguous but also highlights the varying spatial resolution across the wavebands sampled. The red curve on Fig. 1 shows the simple best-SED fit to these data from the MAGPHYS energy balance software of da Cunha, Charlot \& Elbaz (2008). The blue curve shows the actual energy production within this galaxy. In particular it is worth noting the significant change in the slope of the SED in the blue optical region where the observed colour is often erroneously used to divide the galaxy population into intrinsically red and blue populations. Indeed this object is observationally red with a high-Sérsic index yet also dusty and star-forming (i.e., intrinsically blue). This system alone highlights that galaxies do not simply come in two flavours but are a medley of intermingled parameters with all possibilities existing in colour/star-formation/profileshape/opacity/halo/stellar mass parameter space. In order to complete this analysis not only are broad spectrum measurements vital but in the case of non-detections robust upper limits with meaningful errors. This demands the definition of the sample in a single band from which measurements/upper-limits are garnered across the full electromagnetic range. A key question is which band to start from, possibly the most logical is that most closely aligned to stellar mass, i.e., $H$ or $K$ band. 


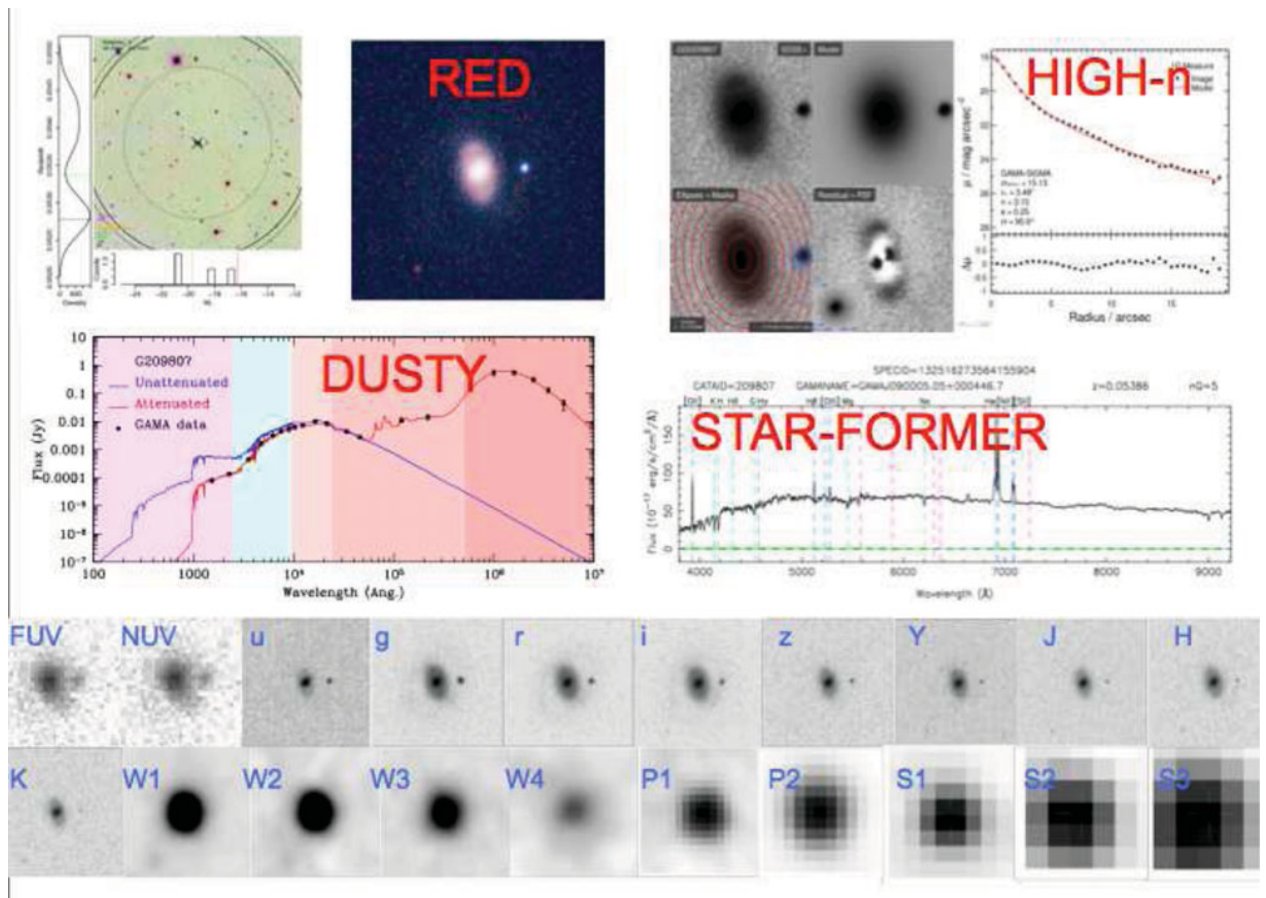

Figure 2. The information available for our 380,000 galaxies. Which includes (clockwise from top-right): halo and group information, colours, 2D profiles (single Sérsic and bulge-disc decompositions), spectroscopic line analysis, panchromatic imaging, and UV-farIR SED modelling.

\section{Summary}

Unlocking the interdependency of galaxy properties as a function of environment and redshift requires a comprehensive panchromatic survey such as GAMA. Fig. 2 shows the type of data we are now assembling for 380,000 galaxies which will enable us to move beyond simple two-dimensional descriptions of the galaxy population and allow us to explore the rich interdependencies of the many properties which we will measure. Extensions to fainter flux-limits, higher spatial resolutions, and higher redshifts are underway.

\section{References}

Baldry I. K., et al., 2010, MNRAS, 404, 86

Baldry I. K., et al., 2012, MNRAS, 421, 621

da Cunha E., Charlot S., \& Elbaz, D., 2008, MNRAS, 388, 1595

Driver S. P., et al., 2009, $A \& G, 50,12$

Driver S. P., et al., 2011, MNRAS, 413, 971

Driver S. P., et al., 2012a, MNRAS, 427, 3244

Driver S. P., et al., 2012b, Panchromatic properties of galaxies in wide-field optical spectroscopic and photometric surveys, IAU Symposium 284, p268-278

Gunawardhana M., et al., 2013, in press

Kelvin L. S., et al., 2012, MNRAS, 421, 1007

Lopez-Cruz M., et al., 2013, in press

Loveday J., et al., 2012, MNRAS, 420, 1239

Robotham A. S. G., et al., 2010, PASA, 27. 76

Robotham A. S. G., et al., 2011, MNRAS, 416, 2640

Robotham A. S. G., et al., 2011, MNRAS, in press

Taylor E., et al., 2011, MNRAS, 418, 1587

Taylor E., et al., 2013, MNRAS, in press 